\title{
LA RECEPCIÓN DE LOS TEXTOS DE DARWIN Y WALLACE EN LA LINNEAN SOCIETY. UNA CRÓNICA DEL SILENCIO
}

\section{DARWIN'S AND WALLACE'S THEORIES AND THEIR RECEPTION IN THE LINNEAN SOCIETY OF LONDON. ALL QUIET IN THE SCIENTIFIC FRONT}

\author{
José Luis GonZÁlez ReCIO \\ Departamento de Filosofía I. Facultad de Filosofía \\ Universidad Complutense de Madrid
}

RESUMEN: La lectura en la Linnean Society de los textos con los que Darwin y Wallace daban a conocer sus tesis transformistas ha sido objeto de dos interpretaciones historiográficas muy diferentes. Para algunos, fue un momento revolucionario y excepcional en la historia de la ciencia, cuya fecha abre el inicio de la biología moderna. De acuerdo con otras percepciones de lo ocurrido aquel día, los ensayos de ambos naturalistas pasaron poco menos que inadvertidos y no fueron valorados según merecían. El presente trabajo procura descender a los detalles, con la intención de ofrecer un análisis más ecuánime y preciso de lo ocurrido.

PALABRAS ClaVE: Selección natural, historia de la biología, Darwin, Wallace.

ABSTRACT: The reading of Darwin and Wallace's texts at the Linnean Society posing the authors' theses on transformism has been accounted for by two very different historiographical interpretations. For some, such an event meant a revolutionary and exceptional milestone in the history of 
science, a moment that signals the beginning of modern biology. According to other perceptions of what happened that day, the essays by both naturalists went pretty much unnoticed and were not given the credit that they deserved. The present paper intends to get a closer look at the details, aiming to offer a more balanced and precise analysis of the events.

KEYWORDS: Natural selection, history of biology, Darwin, Wallace.

\section{Los tres documentos}

Londres, tarde del 1 de julio de 1858. Thomas Bell, presidente de la Linnean Society, concede la palabra al secretario, John Joseph Bennett, quien inicia la lectura de las comunicaciones presentadas a la reunión extraordinaria de la sociedad. La última sesión ordinaria debería haberse celebrado el 17 de junio, pero el fallecimiento del insigne Robert Brown — vicepresidente de la institución- ha obligado a posponerla hasta el 1 de julio. Respetando el orden del día, con anterioridad se ha dado cuenta de las donaciones recibidas para el museo y la biblioteca ${ }^{1}$, se ha procedido a la elección del nuevo vicepresidente — cargo que pasaría a ocupar George Bentham ${ }^{2}-$ y, por último, se han leído unas páginas en memoria del naturalista desaparecido, en las que no han dejado de recordarse sus

1 La relación de donaciones incluía: las Mémoires de la Society Royale des Sciences de Liège, el Journal of the Royal Society, los Abstracts of the Proceedings of the Geological Society, The Philosophical Magazine, y los Annals of Natural History, presentados por Richard Taylor; The Literary Gazette, presentada por Lovell Reeve; una colección de dibujos originales de orquídeas de Tasmania de William Archer, presentada por el señor Archer; The Handbook of British Flora de George Bentham; The Grasses of Great Britain (parte 9), presentado por J. E. Sowerby; Examination of Pavon's collection of Peruvian Barks contained in the British Museum, presentado por su autor, el señor J. E. Howard; Flora Melitensis, presentada por el Dr. Giovanni Carlo Grech Delicata; y especímenes of Highgate Resin, presentados por J. W. Netherall. Véase MoodY, J.W.T., 1971, «The reading of the Darwin and Wallace papers: an historical "non event" ", Journal of the Society for the Bibliography of Natural History 5(6): 474.

${ }_{2}$ Nombrar al sustituto de Brown era el motivo central de aquel encuentro. La elección de Bentham - sobrino de Jeremy Bentham-, con una biografía en la que destacaban sus estudios sobre Botánica, Lógica y Jurisprudencia, fue anunciada por el presidente tras el escrutinio de los votos que llevaron a cabo Berthold Seemann, William Archer y Robert Heward. Véase L.c., pp. 474-475. 
sesenta años de vinculación a la Sociedad Linneana. Concluidas las anteriores formalidades, comienza la lectura de las diferentes contribuciones. La primera, escrita por Charles Robert Darwin y Alfred Russel Wallace, había llegado a las manos de Bennett sólo un día antes, con una carta de presentación que habían redactado Sir Charles Lyell y el doctor Joseph Dalton Hooker en los siguientes términos ${ }^{3}$ :

«Mi querido Señor:

Los documentos adjuntos que tenemos el honor de trasladar a la Linnean Society y que se refieren a la misma cuestión, a saber, las leyes que afectan a la producción de variedades, razas y especies, contienen los resultados de las investigaciones de dos infatigables naturalistas, Mr. Charles Darwin y Mr. Alfred Wallace».

Habiendo concebido estos caballeros, de manera independiente ${ }^{4}$, justo la misma y muy ingeniosa teoría para explicar la aparición y perpetuación de las variedades y las formas específicas sobre nuestro planeta, pueden reclamar ambos honradamente el mérito de ser pensadores originales en esta línea de investigación. Al no haber publicado ninguno de ellos sus puntos de vista - pese a que hemos instado repetidamente a Mr. Darwin durante muchos años para que lo hiciera-, y dado que ambos autores han puesto ahora en nuestras manos sus textos sin reserva alguna, creemos que promovería del mejor modo posible los intereses de la Ciencia el que una selección de los mismos sea presentada ante la Linnean Societyn's.

3 Bennett leyó la carta antes de reproducir el contenido de los textos de Darwin y Wallace.

${ }^{4}$ Los dos naturalistas se habían conocido a comienzos de 1854 en el Museo Británico, al que Wallace acudió con frecuencia antes de partir en una expedición hacia el archipiélago malayo, con la intención de recoger datos en el museo sobre las especies más raras de aves, escarabajos y mariposas que debería encontrar en las islas. Así lo recordaba el propio Wallace cuando escribía a Alfred Newton el 3 de diciembre de 1887: «I had hardly heard of Darwin before going to the East, except as connected with the voyage of the Beagle which I think I had read. I saw him once for a few minutes in the British Museum before I sailed». Carta recogida en MARCHAND, J. , 1916. Alfred Russel Wallace: Letters and Reminiscences, London-New York-Toronto-Melbourne: Cassell \& Company, 2005, p. 105.

5 Los documentos fueron publicados en el Journal of the Proceedings of the Linnean Society, Zoology, 3: 45-62, en agosto de 1858. La carta de Lyell y Hooker se reproduce entre las páginas 45 y 46. 
La carta enumera a continuación los documentos aportados, que consistían en los extractos de un trabajo manuscrito sobre las especies compuesto por Darwin en $1844^{6}$, el resumen de una carta privada que había dirigido al profesor Asa Gray en septiembre de $1857^{7}$ y un ensayo de Wallace titulado «Sobre la tendencia de las variedades a separarse indefinidamente del tipo original ${ }^{8}$. Los socios escucharon en primer lugar, pues, el resumen enviado por Darwin «Sobre la variación de los seres orgánicos en estado de naturaleza, sobre los medios naturales de selección y sobre la comparación de las razas domésticas y las verdaderas especies»". Empezaba así: «De Candolle ha declarado en un elocuente pasaje que toda la naturaleza está en guerra, de los organismos entre sí o de éstos con el mundo exterior. Contemplando su faz apacible, cabría inicialmente ponerlo en duda,

${ }^{6}$ Hooker y Lyell precisaban sobre dicho trabajo que «was sketched in 1839 , and copied in 1844 , when the copy was read by Dr. Hooker, and its contents afterwards communicated to Sir Charles Lyell» (Charles Darwin, Esq., F.R.S., F.L.S.., \& F.G.S., and Alfred Wallace, Esq. Communicated by Sir Charles Lyell, F.R.S., F.L.S., and J.D. Hooker, Esq., M.D., V.P.R.S., F.L.S., \&c., 1858, "On the Tendency of Species to form Varieties; and on the Perpetuation of Varieties and Species by Natural Means of Selection", Journal of the Proceedings of the Linnean Society, Zoology, 3: 45). La noche del 29 de junio, Darwin escribió a Hooker tras leer una carta en la que éste le apremiaba para que enviase los documentos: «I have just read your letter, and see you want the papers at once. I am quite prostrated, and can do nothing, but I send Wallace, and the abstract of my letter to Asa Gray, which gives most imperfectly only the means of change, and does not touch on reasons for believing that species do change. I daresay all is too late. I hardly care about it. But you are too generous to sacrifice so much time and kindness. It is most generous, most kind. I send my sketch of 1844 solely that you may see by your own handwriting that you did read it». La carta está recogida en DARWIN, F. (ed.) 1887. The life and letters of Charles Darwin, including an autobiographical chapter, London: John Murray, vol. 2, pp. 119-120. Darwin conservaba el resumen que había enviado a Hooker con las anotaciones que éste hizo de su puño y letra tras haberlo leído.

7 "...in which he repeats his views, and which shows that these remained unaltered from 1839 to 1857». Journal of the Proceedings of the Linnean Society, Zoology, 3: 45.

${ }^{8}$ La carta de Lyell y Hooker explicaba: «This was written at Ternate in February 1858, for the perusal of his friend and correspondent Mr. Darwin, and sent to him with the expressed wish that it should be forwarded to Sir Charles Lyell, if Mr. Darwin thought it sufficiently novel and interesting. So highly did Mr. Darwin appreciate the value of the views therein set forth, that he proposed in a letter to Sir Charles Lyell, to obtain Mr. Wallace's consent to allow the Essay to be published as soon as possible." Ibid.

9 En el diario de sesiones quedó recogido con el siguiente título completo: «Extract from an unpublished Work on Species, by C. Darwin, Esq., consisting of a portion of a Chapter entitled, "On the Variation of Organic Beings in a state of Nature; on the Natural Means of Selection; on the Comparison of Domestic Races and true Species"”, Journal of the Proceedings of the Linnean Society, Zoology, 3: 46-50. 
pero la reflexión probará inevitablemente que es cierto. La guerra, sin embargo, no es constante sino recurrente: guerra, en pequeño grado, durante cortos períodos, y más severa en períodos ocasionales distantes; de aquí que sus efectos sean pasados por alto con facilidad $»^{10}$. Añadía Darwin de inmediato que se trata de la doctrina de Malthus aplicada con una fuerza mucho mayor, puesto que la restricción moral que en algún grado existe por lo que se refiere a la reproducción de los seres humanos está por completo ausente en el caso de los animales. Al carecer éstos de medios artificiales para nutrirse, cuentan por lo general con una cantidad de alimentos constante, mientras que el incremento de todos los organismos tiende a ser geométrico. Siguen algunos ejemplos sobre el aumento desmesurado de los ratones en La Plata, la introducción del caballo en Sudamérica o los cálculos que pueden hacerse en torno a la descendencia de las aves. En todos los casos la conclusión es la misma: debemos pensar en el enorme poder multiplicador que anualmente actúa dentro de todos los animales o en las incontables semillas que son dispersadas a través de cientos de mecanismos naturales, a la vez que nos vemos obligados a reconocer que el porcentaje medio de los habitantes de una región permanece constante. Hemos de tener en mente, así, que este promedio de individuos sobrevive como resultado de una lucha repetida con otras especies, con las condiciones externas de vida, y que cada individuo de cualquier especie mantiene su lugar en la naturaleza por su propia lucha para adquirir alimento o por la lucha de sus padres con otros individuos de la misma o de diferente especie. Ahora bien, imaginemos que las condiciones externas características de una zona geográfica sufrieran una ligera alteración. En ese caso las proporciones relativas de los habitantes de dicha zona se modificarían levemente. Pero si el número de habitantes fuese pequeño, el libre acceso desde otros lugares fuera posible y el cambio en las condiciones continuase progresando, entonces los habitantes originarios dejarían de estar adaptados a tales condiciones cambiantes. ¿Cabe dudar, en ese caso, — se preguntaba Darwin-que la lucha de cada individuo para subsistir depende de aquellas diminutas modificaciones en su estructura, hábitos o instintos que lo adapten mejor a las nuevas condiciones? Al mismo tiempo, aquellos de sus descendientes que heredasen tal variación — por pequeña que fuera — tendrían también más oportunidades de mantenerse vivos. Cada año nacen más individuos de los que pueden sobrevivir, por lo que el más pequeño grano en la balanza decidirá cuáles perecerán y cuá-

${ }^{10}$ L.c., p. 46. 
les no. Dejemos actuar a este poder de selección y a la muerte durante unos miles de generaciones: ¿quién se atreverá a firmar que no tendrán ningún efecto?

Junto a ese medio natural de selección, por el que los animales o plantas son preservados, en las especies con dos sexos existe además otro agente que tiende a producir el mismo efecto: la lucha de los machos por las hembras. Es, en general, una lucha sometida a las leyes de cualquier batalla, salvo en el caso de las aves, entre las que la victoria depende de la gracia de su canto, de su belleza o de la eficacia de su cortejo. Los machos más vigorosos y sanos, con una perfecta adaptación, serán habitualmente los que ganen semejantes combates. Este tipo de selección es, no obstante, menos riguroso que el otro. No requiere la muerte de los que tienen menos éxito, puesto que sólo da lugar a una menor descendencia. Es más, la lucha se produce en una época del año en la que los alimentos son abundantes, y quizá el efecto producido sea la modificación de los caracteres sexuales secundarios, que no están relacionados con la capacidad para obtener comida o para vencer a potenciales enemigos sino para luchar o rivalizar con otros machos. El texto concluía con una comparación: «el resultado de esta lucha entre los animales machos puede equipararse en algunos aspectos con el que logran aquellos criadores que prestan poca atención a la selección cuidadosa de todos sus animales jóvenes y más al uso ocasional de un emparejamiento selecto» ${ }^{11}$.

Se procedió después a la lectura de algunos pasajes de la carta que Darwin había enviado a Asa Gray en septiembre de $1857^{12}$. El resumen reproducido comenzaba con unos comentarios sobre la selección artificial. En ellos se aludía a la maravillosa capacidad transformadora de la selección llevada a cabo por el hombre. Dicha selección había sido efectuada de una manera metódica en Europa sólo durante el último medio siglo, pero tuvo lugar desde los tiempos más

11 L.c., p. 50.

12 Abstract of a Letter from C. Darwin, Esq., to Prof. Asa Gray, Boston, U.S., dated Down, September 5th , 1857. La carta está catalogada con el número 2.136 en el monumental proyecto on line The Correspondence of Charles Darwin, reproducción digital de la edición preparada por Burkhardt, F., Smith, S. Porter, D. et al. (eds.), 1985-2009. The correspondence of Charles Darwin. Cambridge: Cambridge University Press. Aparece concretamente en el volumen 6 de 1990 , que incluye cartas de los años 1856 y 1857 . Puede consultarse en http://www.darwinproject.ac.uk/ darwinletters/calendar/entry-2136.html. Había sido ya publicada en DARWIN, F. (ed.), 1887. The life and letters of Charles Darwin, including an autobiographical chapter, London: John Murray, vol. 2, pp. $120-125$. 
remotos. Estaba claro que la selección intencional constituía el principal agente en la producción de las razas domésticas. Una selección que actúa sólo por la suma de variaciones más o menos pequeñas, ocasionadas por las condiciones externas o por el mero hecho de que en la generación cada hijo no es absolutamente similar a sus progenitores. Si suponemos ahora que alguien no sólo juzgase por las apariencias externas sino que pudiera estudiar la totalidad de la organización interna, y que persiguiera un objetivo durante millones de generaciones, ¿qué no sería capaz de conseguir? — se preguntaba Darwin. En el estado de naturaleza tienen lugar variaciones en todas las partes de la organización, de forma que la selección opera de un modo certero, preservando aquello que es mejor para cada ser vivo. Hay que tener en cuenta que la completa superficie de la Tierra sería incapaz de mantener la descendencia que una pareja de animales llegaría a tener en unos cientos de años. Sin embargo, sólo unos pocos de los nacidos anualmente son capaces de mantenerse con vida y propagar su clase. De esta manera, la más insignificante diferencia determina quién sobrevivirá y quién perecerá. A su vez, las variedades así constituidas coexistirán con sus formas madres o con frecuencia las exterminarán. Darwin era consciente de las dificultades que su teoría planteaba, mas sugería a Asa Gray que en su mayor parte quedarían resueltas por la evidencia de que natura non facit saltum, por la lentitud de los cambios y por la extrema imperfección del registro geológico. La carta terminaba con unas consideraciones sobre el principio de divergencia, juzgado importante en relación con el origen de las especies. Al reproducirse rápidamente, todo ser orgánico se esfuerza por incrementar el número de sus vástagos. Lo mismo puede decirse de la progenie de cualquier especie: tras haberse diversificado en variedades, subespecies o especies verdaderas, intentará colonizar tantos y tan diversos lugares en la economía de la naturaleza como sea posible ${ }^{13}$.

El último texto leído, como ya he dicho, fue el trabajo de Wallace «Sobre la tendencia de las variedades a separarse del tipo original». Era el documento más extenso de los tres aportados. Comenzaba con un comentario en torno a la supuesta tendencia de las variedades domésticas a regresar hacia la forma normal de la

${ }^{13}$ Darwin enunciaba, finalmente, el significado taxonómico y filogenético de las ideas expuestas: «This I believe to be the origin of the classification and affinities of organic beings at all times; for organic beings always seem to branch and sub-branch like the limbs of a tree from a common trunk, the flourishing and diverging twigs destroying the less vigorous-the dead and lost branches rudely representing extinct genera and families». L.c., p. 53. 
especie de la que procedían. Wallace argumentaba, sin embargo, que no existían datos fiables sobre la manera en que las variedades se producen en estado salvaje y que, de este modo, el gran valor concedido por los naturalistas a la estabilidad observada en el estado doméstico, como prueba de la inmutabilidad de las especies, carecía de justificación ${ }^{14}$. Es cierto — continuaba— que se cree en la realidad de las llamadas «variedades permanentes o verdaderas» — razas de animales con una manifiesta estabilidad y que difieren tan poco de otras, que no es posible determinar cuál procede de cuál一, mas tal hecho, aparentemente incompatible con la invariabilidad de las especies, se desprecia al asumirse que son variedades con límites estrictos y que no pueden alejarse del tipo original, pero sí retornar a él según ocurre en estado doméstico. Luego, el texto enunciaba directamente la meta en él perseguida, que no era sino mostrar la presencia de un principio general en la naturaleza capaz de generar la aparición de variedades que se alejan más y más de sus especies madre, y de hacer que en el estado doméstico se produzca una tendencia a la reversión. En este punto, Wallace introducía la idea de lucha por la existencia ${ }^{15}$, pues, en efecto, la vida de los animales salvajes consiste en esa contienda permanente. Su existencia y la de su prole depende del pleno ejercicio de las facultades y energías que poseen. La posibilidad de conseguir alimento en las estaciones menos favorables y de escapar a los ataques de sus enemigos más peligrosos son las condiciones primarias que determinan la vida tanto de los individuos como de las especies. Dichas condiciones fijan también el número de individuos que compone una especie, por lo que mediante una consideración cuidadosa de todas las circunstancias — sugería el naturalista galés— se podrá comprender y explicar en alguna medida lo que a primera vista parece tan inexplicable: la excesiva abundancia de algunas especies, mientras que otras, estrechamente ligadas a ellas, sean tan raras. ¿Por qué — se pregunta poco después - son las aves acuáticas y en especial las aves marinas tan numerosas? No porque sean más prolíficas que otras sino porque generalmente no les falta alimento. En sentido inverso, los gatos salvajes se reproducen con facilidad y tienen pocos enemigos: ¿por qué entonces no son nunca tan abundantes como los conejos? La única respuesta inteligible es que el aporte de comida con el que cuentan resulta precario. De acuerdo con el criterio de Wallace, parecía evidente, así, que en tanto las condiciones físicas de una región permanecen inaltera-

\footnotetext{
14 Véase, asimismo, l.c., p. 53.

15 "The life of wild animals is a struggle for existence». Ibid.
} 
das, su población animal no puede crecer. Si el número de individuos de una especie crece, por fuerza ha de disminuir el número de individuos de aquellas otras que comparten el mismo tipo de alimentación. Una gran cantidad de seres vivos ha de morir cada año. Como la supervivencia de cada animal depende de él mismo, perecerán los más débiles — los jóvenes, los de más edad, los enfermos-, mientras que prolongarán su existencia los más vigorosos y de mejor salud; aquellos capaces de obtener alimento regularmente y de evitar a sus numerosos enemigos. Lo que tiene lugar, en suma, es una lucha por la existencia en la que siempre sucumbirán los más vulnerables.

Una vez establecidos estos hechos — que la población animal de una región es generalmente estacionaria y que la abundancia o escasez de individuos de una especie es debida por entero a su organización y hábitos- estamos en condiciones de examinar lo que ocurre con las variedades. Cualquier variación en la forma típica de una especie ha de tener un efecto definido sobre los hábitos y capacidades de los individuos que la componen. El mero cambio de color podría afectar a su seguridad, al hacerlos más fácilmente localizables. Un mayor o menor crecimiento de su pelo haría que sus hábitos cambiaran. Y modificaciones más importantes, como el aumento de la fuerza o el tamaño de sus miembros, repercutirán en su modo de alimentación o en las dimensiones del territorio que podrían habitar. Es evidente — señalaba Wallace - que las modificaciones prolongarán o acortarán la existencia de quienes las sufran. Un antílope con patas más cortas o débiles estará más expuesto a los ataques de los carnívoros; y una paloma con alas menos poderosas tarde o temprano encontrará dificultades para obtener alimento. En ambos casos el resultado será un descenso del número de individuos que presentan la variación. Por el contrario, cualquier variedad que haya incrementado sus medios de subsistencia verá crecer como efecto el número de sus individuos. Todas las variedades pueden clasificarse, así, en dos clases: aquellas que bajo las mismas condiciones nunca alcanzarán la población de la especie de la que proceden; y aquellas otras que con el tiempo lograrán una población superior. Si resultase, además, que en la zona habitada se produjera una alteración de las condiciones físicas, es evidente que las variedades más débiles y peor organizadas se verían amenazadas y que se extinguirían si la presión se hiciese más severa. A su vez, las variedades superiores quedarán preservadas y ocuparán el lugar de las especies o variedades extinguidas, en caso de que vuelvan a darse condiciones físicas más favorables. Por todo ello, las nuevas variedades reemplazarán a las especies madre, de las que serán formas más perfectamente desa- 
rrolladas y organizadas. No podrán sufrir una reversión hacia las formas originales, puesto que éstas son inferiores e incapaces de competir por la existencia. Ahora bien, semejantes razas o variedades nuevas darán lugar en un futuro a otras que se convertirán en dominantes mediante la misma ley general, por lo que, en el estado de naturaleza, hemos de admitir como resultado un proceso de divergencia continua a partir del simple hecho del nacimiento de variedades.

Wallace quería, además, señalar las diferencias respecto a lo que ocurre en domesticidad. El asunto tenía su importancia, porque la reversión hacia los tipos originales en el estado doméstico se empleaba con frecuencia como argumento a favor de la inmutabilidad de las especies. Darwin creyó que la proyección analógica al estado salvaje de la creación de razas domésticas tenía un valor metodológico que debía aprovecharse ${ }^{16}$. Wallace, por su parte, prefería marcar las diferencias entre uno y otro ámbito, explicando que la reversión observada por los agricultores y criadores podía entenderse bien, precisamente como consecuencia de la hipótesis propuesta. La diferencia esencial entre los animales salvajes y los domésticos estriba en que la supervivencia de los primeros depende por completo del pleno uso de sus capacidades físicas y de sus sentidos, mientras que los segundos sólo los ejercitan parcialmente o incluso no los emplean en absoluto. Un animal salvaje tiene que buscar sus alimentos y luchar por ellos; debe ejercitar su vista, su oído su olfato, encontrar refugio ante las inclemencias de las estaciones; ha de trabajar por la subsistencia y la seguridad de su prole; no hay músculo de su cuerpo cuya actividad no sea requerida diariamente; no existe sentido o facultad que no resulte fortalecida por su utilización continua. Muy al contrario, el animal doméstico tiene su alimento asegurado, está protegido contra los cambios climáticos y los ataques de sus enemigos naturales; no emplea ni la mitad de sus sentidos y facultades, y la otra mitad son puestos en acción de una manera limitada. Cuando se presenta una variación en alguno de estos animales, afectando a sus órganos o sentidos, tal modificación es completamente inútil. En el animal salvaje, sin embargo, cualquier cambio debe afectar a la obtención de alimentos, a los hábitos y a la economía general de la especie. Aparece algo así como un animal nuevo con una capacidad de supervivencia incrementada respecto a

16 Sobre la conexión de esta analogía con los recursos metodológicos avalados por John Herschel —el gran astrónomo a quien Darwin tanto admiró-, véase HersCHEL, J., 1830. A Preliminary Discourse on the Study of Natural Philosophy, Chicago-London: The University of Chicago Press, 1987, JS 134-136. 
los animales inferiores con quienes compite. Gran parte de las razas domésticas nunca se habrían desarrollado en el estado de naturaleza. Al tratarse de formas inferiores, con rapidez se habrían extinguido. Si las variedades domésticas fuesen abandonadas en la naturaleza, desaparecerían, ciertamente, o sufrirían una regresión hacia formas próximas a las existentes en el estado salvaje.

El punto crucial - para Wallace- era, por lo tanto, que no cabía hacer inferencias a partir de lo que ocurre con las variedades domésticas, extendiendo las conclusiones al estado de naturaleza ${ }^{17}$. Es más: casi todo lo que puede afirmarse de los animales domésticos no es aplicable a los animales salvajes y viceversa —recalcaba—. La hipótesis de Lamarck según la cual los cambios progresivos en las especies obedecen a los intentos de los animales por desarrollar sus propios órganos, y así modificar su estructura y sus hábitos, es una hipótesis repetida, pero fácilmente refutable. Parece que se ha entendido que la cuestión quedaba con ello zanjada por completo. Y, sin embargo, - Wallace concluía- las ideas de Lamarck resultan innecesarias cuando se comprende que los mismos resultados pueden producirse por la acción de principios que operan en la naturaleza de modo constante. Las últimas palabras del documento — también conocido como el Ternate Paper of 1858, por el lugar donde fue redactado- eran éstas:

«Creemos haber mostrado que en la naturaleza hay una tendencia al cambio progresivo continuo de ciertas clases de variedades que se alejan más y más del tipo original —un cambio progresivo al que no parece que exista razón para poner límites definidos- y que el mismo principio que produce este resultado en un estado de naturaleza explicará también por qué las variedades domésticas tienen una tendencia a regresar al tipo original. Este cambio progresivo - mediante pequeños pasos en diferentes direcciones, pero siempre controlado y equilibrado por las condiciones necesarias, sólo de acuerdo con las cuales la existencia puede ser preservada - creemos que puede producirse de modo que justifique todos los fenómenos presentes en los seres organizados, su extinción y sucesión en las pasadas edades, así como todas las extraordinarias modificaciones de forma, instinto y hábitos que exhiben ${ }^{18}$.

${ }^{17}$ En especial, inferencias sobre la regresión a los tipos o formas originales.

18 Wallace, A.R., 1858. "On the Tendency of Varieties to depart indefinitely from the Original Type», Journal of the Proceedings of the Linnean Society, Zoology, 3: 62. 
Una vez escuchada la comunicación conjunta de Darwin y Wallace, la sesión continuó. En Burlington House (Picadilly), sede de la sociedad, no hubo la menor reacción o comentario. Se dio lectura al siguiente trabajo en la lista preparada al efecto. Eran las "Notes on the organisation of Phoronis hippocrepis» que había presentado el doctor Fredric. D. Dyster. Para algunos —el propio Hooker, Lyell, más tarde Asa Gray o Thomas Huxley y mucho después Julian Huxley, junto a una parte importante de los historiadores de la Biología - se había producido el gran acontecimiento. En la historia de la Ciencia, aquel 1 de julio de 1858 quedaba marcado como un día memorable, como el momento de la presentación solemne y revolucionaria de un transformismo que empezaba a ser maduro. Pocas fechas en el desarrollo del conocimiento científico alcanzarían su significado teórico, cultural y social, pues no siempre la presentación pública de hipótesis con tanto alcance, significado y valor está ligada a un un evento tan definido, tan preciso en el espacio y en el tiempo o tan unido a los usos institucionalizados de las sociedades científicas. Sin embargo, esta visión de lo acaecido en la Linnean Society pone insensiblemente el acento en lo que iba a ocurrir con posterioridad. Es decir, sólo atendiendo a los hechos que tendrían lugar después se ha podido otorgar una significación histórica a lo que sucedió durante las últimas horas de la tarde de aquel día. En realidad, si nos atenemos a lo acontecido dentro de la reunión misma, la respuesta que recibieron los textos de Darwin y Wallace queda resumida en el completo silencio de los asistentes. Ningún reconocimiento; tampoco ninguna observación u objeción. Esta aparente indiferencia hizo que James W.T. Moody — miembro de la sociedad— publicara en 1979 un artículo de gran repercusión entre los historiadores. Se titulaba «The reading of the Darwin and Wallace papers: an historical "non event" ${ }^{19}$. En él defendía que pese a haber sido identificada con el inicio de la revolución darwinista, con el comienzo de la biología moderna o con el principio de una nueva era para el pensamiento científico, la reunión del 1 de julio de 1858 en la Linnean Society merece equipararse, por el contrario, a las que tendrían lugar los días 8 de febrero y 8 de marzo de 1865 en la Sociedad de Historia Natural de Brno, dentro de las cuales Gregor Mendel presentó sus "Experimente auf Pflanzenkreuzung» ${ }^{20}$. En uno y otro caso nos hallamos ante no-acontecimientos históricos, si valoramos la forma en que fueron recibidos los trabajos expuestos ante los socios. Así pues, lo

19 Journal of the Society for the Bibliography of Natural History 5(6): 474-476.

${ }^{20}$ La comunicación sería publicada un año después en las actas de la sociedad como Versuche über Pflanzen-Hybriden (Verhandlungen des naturforschenden Vereines in Brünn, 4: 3-47). 
que ocurrió en aquel encuentro de notables científicos victorianos ha merecido dos lecturas muy diferentes: la que no duda en otorgarle una extraordinaria relevancia y la que diluye su trascendencia hasta casi hacerla desaparecer. Mi intención en estas páginas es analizar lo que hay de cierto en ellas, para ofrecer después una tercera interpretación — creo que más justa y equilibrada- en torno a lo que sucedió aquel día. Como primer paso, tiene interés recordar — pese a ser de sobra conocidos - cuáles fueron los hechos previos que condujeron a la presentación conjunta de los textos de Wallace y Darwin.

\section{1854-1858: el camino hacia lajoint communication}

En 1852, tras su regreso de un viaje a Sudamérica, Wallace alquiló una casa en Upper Albany Street, cercana a los Zoological Gardens de Rgents's Park, donde se dispuso a redactar el relato de su expedición al Amazonas y Río Negro, y a clasificar los especímenes recogidos. El trabajo le llevaría cerca de dieciocho meses, durante los cuales acudió con frecuencia al Museo Británico, para obtener datos sobre la zoología del archipiélago malayo — lugar al que pensaba trasladarse pronto. Como he indicado más arriba, fue en una de estas visitas, a principios de 1854, cuando se produjo su encuentro con Darwin. El siguiente contacto entre ambos tuvo lugar en 1856, a través de Samuel Stevins — agente de Wallace-, quien envió a Darwin un memorándum con observaciones sobre razas domésticas que aquél había realizado en Sarawak (isla de Borneo) ${ }^{21}$. Fue allí donde Wallace comenzó a escribir las notas sobre la distribución geográfica de los animales y las plantas que se convertirán en su trabajo «On the Law which has regulated the Introduction of New Species», en el que se mostraba convencido de que toda especie nace rodeada de especies preexistentes muy próximas. Remitió el artículo a los Annals and Magazine of Natural History ${ }^{22}$, donde fue publicado,

${ }^{21}$ Véase Gardiner, B., 1995. «The Joint Essay of Darwin and Wallace», The Linnean 1995 Volume (11) 1: 13.

22 WallaCe, A.R., 1855. "On the Law which has regulated the Introduction of New Species", Annals and Magazine of Natural History, 16 ( $2^{\text {nd }}$ Series): 184-192. El texto puede consultarse en su integridad y con la paginación original en: http://www.wku.edu/ -smithch/wallace/S020.htm. Al final del mismo, Wallace sostenía: «It has now been shown, though most briefly and imperfectly, how the law that Every species has come into existence coincident both in time and space with a pre-existing closely allied species, connects together and renders intelligible a vast number of independent and hitherto unexplained facts. The natural system of arrangement of organic beings, 
despertando un vivo interés entre otros en Charles Lyell. Durante una visita a la residencia de Darwin en Down, que tuvo lugar en abril de 1856, Lyell recomendó a su anfitrión que no dejara de leerlo; sugerencia que Darwin ya había recibido de Edwuard Blyth en una carta que éste le dirigió desde Calcuta el 8 de diciembre del año anterior ${ }^{23}$. Wallace permaneció en Borneo durantes quince meses. Hacia primeros de septiembre de 1856 llegó a la isla de Célebes. Allí escribió su primera carta a Darwin unas semanas más tarde —el 10 de octubre-. Es una carta que no se conserva, por lo que hemos de reconstruir su contenido a partir de la respuesta de Darwin, enviada el 1 de mayo de 1857. En ella explicaba a Wallace que estaba de acuerdo con su indicación sobre el carácter singular de las variedades domésticas frente a las que se producen en estado de naturaleza; apoyaba también la idea de que el clima juega un papel muy limitado como origen de las variaciones y aseguraba a su compatriota que, a la vista de su carta y del artículo que éste había publicado en los Annals, era patente que pensaban de forma muy parecida y que en términos generales compartían las mismas conclusiones. Añadía, asimismo, que hacía veinte años que había empezado a escribir su primer cuaderno sobre la forma en que las variedades y las especies difieren entre sí. Al mismo tiempo, le comunicaba que estaba escribiendo una obra sobre el asunto, si bien tardaría por lo menos dos años en publicarla ${ }^{24}$. Wallace leyó la carta de Darwin en Macassar a finales del verano de 1857, tras haber visitado las

their geographical distribution, their geological sequence, the phænomena of representative and substituted groups in all their modifications, and the most singular peculiarities of anatomical structure, are all explained and illustrated by it...». (L.c., p. 192).

${ }^{23}$ La carta tiene el número 1.792 en la edición de BurkHardT, F., SMITH, S. PORTER, D. et al. (eds.), o.c. vol. 5, 1988. Blyth, especialista en zoología de la India, advierte a Darwin: «Wallace has, I think, put the matter well; and according to his theory, the various domestic races of animals have been fairly developed into species».

$24 « \mathrm{I}$ am much obliged for your letter of Oct. $10^{\text {th }}$. from Celebes received a few days ago: in a laborious undertaking sympathy is a valuable \& real encouragement. By your letter \& even still more by your paper in Annals, a year or more ago, I can plainly see that we have thought much alike \& to a certain extent have come to similar conclusions. In regard to the Paper in Annals, I agree to the truth of almost every word of your paper; \& I daresay that you will agree with me that it is very rare to find oneself agreeing pretty closely with any theoretical paper; for it is lamentable how each man draws his own different conclusions from the very same fact.

This summer will make the $20^{\text {th }}$ year (!) since I opened my first-note-book, on the question how $\&$ in what way do species $\&$ varieties differ from each other. I am now preparing my work for publication, but I find the subject so very large, that though I have written many chapters, I do not suppose I shall go to press for two years». Carta con el número2.086 en BURKHARDT, F., SMITH, S. Porter, D. et al. (eds.), o.c., vol. 6, 1990. 
Islas Aru, y se apresuró a contestar al naturalista de Down el 27 de septiembre de 1857. La carta no se conserva en su integridad, pero sí algunos de sus párrafos más reveladores. En uno de ellos, Wallace se congratula de que Darwin respalde la forma en que concibe la sucesión de las especies. En otro, se queja del poco interés que ha despertado su artículo en los Annals, reconociendo, no obstante, que el trabajo recogía sólo el planteamiento preliminar de una teoría cuya formulación está preparando y tiene ya en parte escrita ${ }^{25}$. Llegado el mes de diciembre, embarcó hacia Ambon, desde donde, el 4 de enero de 1858, escribe a su amigo el entomólogo Henry Walter Bates diciéndole:

«"La sucesión de las especies" no aparecerá tan clara como lo es para ti. Ese trabajo es, por supuesto, simplemente el anuncio de la teoría, no su desarrollo. He preparado un plan y he escrito partes de una obra que abarca la totalidad del tema. Me he esforzado en probar con todo detalle lo que hasta ahora solo había sugerido. Me siento muy gratificado por una carta de Darwin en la que dice que está de acuerdo con casi cada palabra de mi artículo. Él prepara ahora su gran libro «Sobre las especies y las variedades», para el que ha reunido materiales durante veinte años ${ }^{26}$.

La carta fue concluida en Ternate —el 25 de enero de 1858 - e incluía una relación de las distintas especies de insectos que Wallace había podido recoger en las Islas Aru. Unas semanas después —entrado ya el mes de febrero, sin que podamos precisar si se encontraba aún en Ternate o en Gilolo-, mientras decidía cuál iba a ser el próximo destino en su exploración, la malaria le obligó a permanecer en reposo y, a la vez, le dio la oportunidad de meditar sobre uno de los problemas que más le interesaban: el origen de las variedades y la especiación. Dentro de sus recuerdos autobiográficos, escribe años más tarde: «un día algo trajo a mi mente los Principios de la Población (sic) de Malthus, que había leído doce años antes. Pensé en su clara exposición en torno a los obstáculos que impiden el incremento de las poblaciones - la enfermedad, los accidentes, la guerra, el hambre- y que hacen que la población media sea menor en las razas salvajes frente a la que se da en las comunidades civilizadas ${ }^{27}$. Añade, luego, que enton-

\footnotetext{
25 Véase la carta 2.145 en el mismo volumen de la edición de BURKHARDT.

${ }^{26}$ La carta está incluida en MARCHAND, J. , 1916. Alfred Russel Wallace: Letters and Reminiscences, London-New York-Toronto-Melbourne: Cassell \& Company, 2005, vol. 1, pp. 66-67.

27 Wallace, A.R., 1905. My Life: A Record of Events and Opinions, Whitefish (Montana): Kessinger Publishing, 2004, p. 190.
} 
ces se le ocurrió que estas causas o causas similares actúan también continuamente sobre los animales. Dado que éstos se reproducen con mucha mayor velocidad que los seres humanos, la destrucción que tales causas deben producir cada año ha de ser enorme, teniendo en cuenta que el número de individuos de las diferentes especies suele mantenerse estable. Fue así como llegó a preguntarse: "¿por qué unos mueren y otros sobreviven? La respuesta era claramente que en conjunto sobreviven los mejor adaptados $»^{28}$. Siguen a continuación una serie de reflexiones sobre la mejora que este proceso ciego ha de producir en las razas, variedades y especies. Las pequeñas variaciones individuales que resulten ventajosas frente a los lentos cambios del entorno se incorporarán a la organización de cada ser vivo, haciendo que ésta sea la mejor posible. Los que no adquieran esas modificaciones perecerán. Finalmente, declara: «cuanto más lo pensaba, más me convencía de que por fin había encontrado la ley de la naturaleza, tanto tiempo buscada, que resolvía el problema del origen de las especies» ${ }^{29}$.

Wallace había abandonado el fijismo mucho antes. A finales de 1845 así se lo comunica a Bates ${ }^{30}$. Pero lo que ha podido establecer ahora es la ley — según gustaba llamarla - que rige la transformación de los organismos. Darwin, como es sabido, se adelantó. En julio de 1837 había comenzado a escribir su primer cuaderno sobre la transmutación, y quince meses después abrazaba la hipótesis de la selección natural ${ }^{31}$. En su autobiografía lo recuerda así:

«En octubre de 1838, es decir, quince meses después de haber iniciado mi investigación sistemática, leí para entretenerme el ensayo de Malthus Sobre la población. Y al estar bien preparado para reconocer la lucha por la existencia que se produce en todas partes, gracias a la prolongada y continua observación de los hábitos de los animales y las plantas (sic), de inmediato me asaltó la idea de que, bajo estas circunstancias, las variaciones favorables tenderían a ser preservadas y que las desfavorables resultarían destruidas. El

28 Ibid.

29 L.c., p. 191.

30 Véase Gardiner, B., 1995. "The Joint Essay of Darwin and Wallace», The Linnean 1995 Volume (11) 1: 19, cuya descripción de los hechos reproduzco en estos momentos.

31 El proceso puede seguirse en BARret, P.H., GaUTREY, P.J., HARbERT, S., KoHN, D. \& SMITH, S. (eds.), 1987. Charles Darwin's Notebooks, 1836-1844: Geology, Transmutation of Species, Metaphysical Enquiries, Cambridge: Cambridge University Press, London: British Museum (Natural History). 
resultado de ello sería la formación de nuevas especies. Aquí tenía por fin una teoría en la que trabajar. Pero estaba tan preocupado por evitar cualquier prejuicio, que decidí no escribir ni siquiera un esbozo de la misma durante algún tiempo. En junio de 1842 me permití por primera vez la satisfacción de escribir a lápiz un muy breve resumen de 35 páginas de mi teoría; resumen que fue ampliado en el verano de 1844 hasta convertirse en uno de 235 páginas, copiado con todo esmero y que aún conservo» ${ }^{32}$.

Ese mismo año, Darwin mostró el resumen a Lyell y Hooker. Como ya he adelantado $^{33}$, en 1858 lo adjunta a la carta que el 29 de junio envía a éste último, para que recuerde que lo había leído el mismo año en que fue escrito, pues en el original se conservan las observaciones y sugerencias que había hecho a Darwin. 1844 es un año de importancia singular en el desarrollo del evolucionismo darwiniano, en definitiva, y así lo atestigua su siempre citada carta a Hooker del 11 de enero ${ }^{34}$. Lo es también en la historia general del transformismo por la publicación anónima de los Vestiges of the Natural History of Creation, que había escrito Robert Chambers ${ }^{35}$. A pesar de que se tratara de una obra carente de fundamentación científica, su enorme difusión e impacto serviría a Darwin para observar por anticipado qué reacciones debía esperar en el futuro, y conseguiría también que el horizonte de la evolución se abriera ante la sociedad de la época ${ }^{36}$. Por si todo ello fuera poco, no hay que olvidar que la paternidad de la obra fue atribuida por algunos al propio Darwin ${ }^{37}$; un hecho que pone de manifiesto que

32 DARWIn, C.R., 1958. The Autobiography of Charles Darwin 1809-1882. With the Original Omissions Restored. Edited and with appendix and notes by his grand-daughter Nora Barlow, London: Collins, p. 120.

33 Véase la nota número 6.

34 En ella afirma: «At last gleams of light have come, and I am almost convinced (quite contrary to the opinion I started with) that species are not (it is like confessing a murder) immutable». Véase DARWIN, F. (ed.) 1887. The Life and Letters of Charles Darwin, Including an Autobiographical Chapter. London: John Murray, vol. II, p. 23.

35 Chambers, R., 1844. Vestiges of the Natural History of Creation, Chicago: University of Chieago Press: 1994.

36 Sobre la actitud de Darwin, Wallace y Huxley frente a los Vestiges, véase SCHWARTZ, J.S., 1990. "Darwin, Wallace, and Huxley, and Vestiges of the Natural History of Creation», Journal of the History of Biology, 23: 127-153.

37 Véase RuSE, M., 1979. The Darwinian Revolution : Science Red in Tooth and Claw, Chicago: University of Chicago Press, p. 98. La fuente directa es, sin embargo, el propio Darwin. En carta a su primo William Darwin Fox, de 24 de abril de 1845, le dice: «Have you read that stran- 
probablemente su adhesión al transformismo no era absolutamente desconoci$\mathrm{da}$ - pese a la cautela con que el futuro autor de El origen de las especies iría comunicando sus ideas a quienes consideraba confidentes más cercanos, como Hooker, Lyell o Gray-. Es un asunto con cierto interés, pues obliga a matizar la tesis de que la naturaleza de sus textos causó una completa sorpresa en la Linnean Society. Ciertamente, el 1 de julio de 1858 nadie de los asistentes - con excepción de Bell y Bentham - sabía que los resúmenes de Wallace y Darwin iban a ser leídos, pero es muy verosímil que una parte importante de los consagrados naturalistas presentes conociera la inclinación de Darwin hacia las tesis transformistas. Otro tanto puede afirmarse en torno a Wallace, puesto que su artículo en los Annals, según hemos visto, había sido publicado en 1855. Este mismo año marca precisamente el retorno de Darwin al estudio de los problemas relacionados con la transformación. Ha concluido sus obras geológicas, ha supervisado la segunda edición del Journal of Researches, pero, sobre todo, ha dado fin a su impresionante obra sobre los cirrípedos ${ }^{38}$. Vuelve entonces a ocuparse de la distribución geográfica de las plantas. Estudia la capacidad que poseen las semillas para germinar tras ser sumergidas en agua salada, concluyendo que es una propiedad que conservan durante mucho tiempo y que permite conjeturar que pueden ser transportadas a gran distancia por mares y océanos. Los resultados de sus experimentos están contenidos en la primera comunicación que envía a la Linnean Society, precisamente ${ }^{39}$.

ge unphilosophical, but capitally-written book, the Vestiges?: it has made more talk than any work of late, $\&$ has been by some attributed to me.-at which I ought to be much flattered \& unflattered». (Carta 853, en BurkHARdt, F., SMith, S. Porter, D. et al. (eds.), o.c., vol. 3, 1987).

38 1842, The structure and distribution of coral reefs. Being the first part of the geology of the voyage of the Beagle, under the command of Capt. Fitzroy, R.N. during the years 1832 to 1836, London: Smith Elder and Co.; 1844, Geological observations on the volcanic islands visited during the voyage of H.M.S. Beagle, together with some brief notices of the geology of Australia and the Cape of Good Hope. Being the second part of the geology of the voyage of the Beagle, under the command of Capt. Fitzroy, R.N. during the years 1832 to 1836, London: Smith Elder and Co., y 1846, Geological observations on South America. Being the third part of the geology of the voyage of the Beagle, under the command of Capt. Fitzroy, R.N. during the years 1832 to 1836, London: Smith Elder and Co. En 1845, Journal of researches into the natural history and geology of the countries visited during the voyage of H.M.S. Beagle round the worl, London: John Murray. 2d ed. 1851. 1852, A monograph of the sub-class Cirripedia, with figures of all the species. The Lepadide; or, pedunculated cirripedes. London: The Ray Society, Volume 1, y en 1854, A monograph on the sub-class Cirripedia, with figures of all the species. The Balanida, (or sessile cirripedes); the Verrucida, etc. etc. etc. London: The Ray Society. Volume 2.

39 I conclude, under the existing extremely scanty materials for forming any opinion, that some plants might under favourable conditions be transported over arms of the sea 300 or even 
En el mes de abril inicia su correspondencia con Asa Grey ${ }^{40}$. El botánico norteamericano había aludido —en las reseñas que escribió sobre la Flora Japónica de Zuccarini $(1840,1846)$ - al gran número de plantas del oeste de Norteamérica que sólo pueden encontrarse también en el este de Asia. En junio, Darwin vuelve a escribirle y le da a conocer las conclusiones a las que ha llegado sobre el transporte de las semillas y su capacidad para germinar. El intercambio epistolar continúa hasta que el 20 de julio de 1857 da a conocer a Gray su hipótesis sobre las causas de la especiación -asunto que reaparece en la carta del 5 de septiembre, algunos de cuyos párrafos constituyeron el segundo documento aportado a la sesión de la Linnean Society en la que Darwin y Wallace presentaron su comunicación conjunta. Darwin adjuntó a esta carta un resumen de sus ideas, que en esencia era el que había enseñado a Hooker y Lyell en 1844.

El resto de los hechos acaecidos hasta el 1 de julio de 1858 se ha relatado en múltiples lugares. Hago sólo, por consiguiente, un recuento sumario. Tras leer el artículo de Wallace en los Annals, Lyell recomienda a Darwin, a principios de 1856, que ponga por escrito sus ideas del modo más detallado posible. Darwin así comienza a hacerlo, con el objetivo de publicar una voluminosa obra sobre las especies. En ella trabajó regularmente hasta que entre mayo y junio de 1858 recibe una carta que Wallace le dirige desde Ternate, acompañada de un ensayo donde Darwin va a encontrar reflejadas, como en un espejo, sus hipótesis transformistas y el papel que debe otorgarse a la selección natural. La carta se ha perdido. No obstante, podemos imaginar el efecto que produjo en Darwin, acudiendo a la que éste escribió a Lyell el 18 de junio:

«Hace aproximadamente un año me recomendó que leyera un artículo de Wallace en los Annals, que le había interesado; y, puesto que le estaba escribiendo en ese momento, se lo dije, pues sabía que le agradaría. Hoy me ha enviado el documento adjunto y me ha pedido que se lo haga llegar a usted. Creo que merece la pena leerlo. Sus palabras se han hecho realidad, al modo de una venganza para la que debería haber estado preparado. Así

\footnotetext{
more miles in breadth; and if cast on the shore of an island not well stocked with species, might become naturalized. DARWIN, C., 1857. "On the action of sea-water on the germination of seeds». [Leída el 6 de mayo de 1856], Journal of the Proceedings of the Linnean Society of London (Botany) 1: 135.

40 Véase Gardiner, B., 1995. "The Joint Essay of Darwin and Wallace», The Linnean 1995 Volume (11) 1: 20.
} 
me lo dijo usted cuando le expliqué aquí muy brevemente mis puntos de vista sobre la 'selección natural' en términos de la lucha por la existencia. Nunca había visto una coincidencia más asombrosa. Si Wallace tuviera la copia de mi esquema escrito en 1842 no podría haber hecho un resumen mejor! Hasta sus términos aparecen ahora como títulos de mis capítulos.

Por favor, devuélvame el manuscrito. Él no me ha expresado el deseo de que lo publique, pero, por supuesto, escribiré de inmediato ofreciéndolo a alguna revista. De esta forma, mi originalidad, cualquiera que sea, resultará destruida, aunque mi libro, si tuviera algún valor, no se vera perjudicado, pues todo el trabajo consiste en la aplicación de la teoría $»^{41}$.

Como hemos tenido ocasión de ver más arriba, Darwin conocía el contenido del artículo publicado por Wallace en los Annals y había comunicado a éste que sus puntos de vista eran coincidentes. Sin embargo, las cuestiones dirimidas en él se referían al origen de las variaciones individuales y a la comparación entre las variedades domésticas y las que podían producirse en estado de naturaleza. Nada que tuviera que ver aún con el papel de la selección natural, por lo tanto. Mas la nueva carta de Wallace y el manuscrito adjunto entraban directamente en dicha cuestión. Darwin envió a Lyell ese mismo día — 18 de junio- el manuscrito de Wallace. Escribió igualmente a Hooker, para explicarle lo ocurrido y para anunciarle que pensaba renunciar a toda pretensión de prioridad — prioridad a la que Wallace, por cierto, nunca aspiróo ${ }^{42}$. Un año después, contará a éste que había empezado a escribirle, con la intención de anunciarle que no publicaría nada antes de que él lo hiciese. Una carta que no llegó a enviar, al haber reconocido Hooker en su respuesta que, como Lyell, estaba al corriente de las ideas de Darwin desde hacía catorce años ${ }^{43}$. Hooker sugirió entonces una publicación

${ }^{41}$ Carta 2.285, en Burkhardt, F., Smith, S. Porter, D. et al. (eds.), o.c., vol. 7, 1991.

${ }^{42}$ Véase Claridge, M., 2008. "Alfred Russel Wallace - a Welsh Entomologist!», en GARDINER, B., Milner, R. \& Morris, M. (eds.). Survival of the Fittest. A Special Issue of The Linnean celebrating the 150th anniversary of the Darwin-Wallace theory of evolution, Oxford: Wiley-Blackwell, p. 18.

43 "You cannot tell how I admire your spirit, in the manner in which you have taken all that was done about publishing our papers. I had actually written a letter to you, stating that I would not publish anything before you had published. I had not sent that letter to the post when I received one from Lyell and Hooker, urging me to send some MS. to them, and allow them to act as they thought fair and honourably to both of us. I did so». (Carta de Darwin a Wallace del 6 de abril de 1859. Reproducida en MARCHAND, J., 1916. Alfred Russel Wallace: Letters and Reminiscences, London-New York-Toronto-Melbourne: Cassell \& Company, 2005, vol. 1, p. 137. 
conjunta y se comprometió a escribir a Wallace en caso de que Darwin la aceptase. Éste volvió a dirigirse a Lyell los días 25 y 26, preocupado porque alguien pudiera pensar en el futuro - cuando diera a conocer sus ideas - que se había servido de los trabajos de Wallace. En la carta del día 25, le explica que ya en su ensayo de 1844, y más recientemente en su correspondencia con Gray un año antes, están anticipadas las tesis básicas que defiende. Podría probarse, así, que no había tomado nada de Wallace. En la del día 26, confiesa a su amigo que adelantarse ahora a Wallace, tras conocer cómo concibe éste la aparición de nuevas especies, le parece un abuso, con independencia de haber llegado mucho antes a las mismas conclusiones ${ }^{44}$. De cualquier forma, pide a Lyell que si avala su prioridad y está de acuerdo en la presentación simultánea de un resumen de sus tesis y del trabajo de Wallace, escriba directamente a Hooker. El eminente geólogo así lo hizo, con lo que Darwin quedó satisfecho y autorizó la comunicación conjunta. Hooker y Lyell actuaron con toda rapidez. Al haberse suspendido la reunión del 17 de junio en la Linnean Society por la muerte de Robert Brown y no estar prevista otra sesión ordinaria hasta noviembre, era aconsejable aprovechar la convocatoria extraordinaria hecha para el 1 de julio, en la que se elegiría al nuevo vicepresidente. Ni Wallace ni Darwin asistirían a la lectura de sus trabajos. El primero se encontraba en Nueva Guinea ${ }^{45}$. No tuvo ocasión de autorizar el que su ensayo formara parte de la comunicación a la Sociedad Linneana, porque no se le consultó. Darwin estaba pasando por un momento personal de extrema aflicción. El día 28 de junio había perdido a su hijo más pequeño, Charles Waring. En la primera de las dos cartas que dirigió a Hooker el día 29, le da la noticia ${ }^{46}$. El contenido de la segunda, escrita esa noche, ha sido ya comentado ${ }^{47}$. Lo esencial es que hace saber en ella a su más fiel amigo que de inmediato le envía

${ }^{44}$ Véanse las cartas 2.294 y 2.295 en Burkhardt, F., SMith, S. Porter, D. et al. (eds.), o.c., vol. 7, 1991.

45 Sobre los viajes de Wallace en este período y su trayectoría científica hasta que formula la hipótesis de la selección natural, véase SARKAR, S., 2008. "Wallace and Natural Selection, 1858», Resonance: 236-244.

46 «You will, \& so will $\mathrm{M}^{\mathrm{rs}}$ Hooker, be most sorry for us when you hear that poor Baby died yesterday evening. I hope to God he did not suffer so much as he appeared. He became quite suddenly worse. It was Scarlet-Fever. It was the most blessed relief to see his poor little innocent face resume its sweet expression in the sleep of death. Thank God he will never suffer more in this world». (Carta 2.297 del 29 de junio de 1858, recogida en BurkHARDT, F., SMITH, S. PORTER, D. et al. (eds.), o.c., vol. 7, 1991).

47 Véase nota número 6. 
los documentos solicitados. Un día más tarde estarán ya en poder del secretario de la Linnean Society.

\section{La reunión en Burlington House}

Habíamos abandonado la sesión en el momento en que Bennett concluyó la lectura de los textos de Darwin y Wallace. Pese a tratarse de una convocatoria extraordinaria, se decidió también admitir en ella la presentación de algunas comunicaciones programadas para el 4 de noviembre o que deberían haberse trasladado a esa fecha, puesto que llegaron el mismo 1 de julio. Finalmente, el programa dio acogida —en este orden y tras los de Wallace y Darwin- a los siguientes trabajos: las "Notes on the organisation of Phoronis hippocrepis», de F. D. Dyster; las "Observations on Ammocaetus", de Samuel Highley; "On Hanburia, a new genus of Cucurbitacea», de Berthold Seemann; una memoria manuscrita del profesor Pavon, titulada "Nueva Quinologia», con observaciones de J. E. Howard; y dos cartas "on the Vegetation of Angola», del doctor F. Welwitsch, dirigidas a W.W. Saunders. Las «Notes on British Botany», de George Bentham, se habían hecho llegar a Bennett el 1 de julio, pero, como explicaré más tarde, los asistentes no pudieron escucharlas ${ }^{48}$. En opinión de James Moody, los textos de Wallace y Darwin quedaron sepultados bajo semejante cantidad de estudios, cuestiones e intereses. Más que impresionar a los miembros de la sociedad, acabaron perdidos entre la abrumadora cantidad de información que éstos recibieron. Resultó, así, que el concepto de selección natural sobrevoló las cabezas de los asistentes, sin que llegara a posarse en ninguna ${ }^{49}$. En resumen, habría que entender — si es que aceptamos esta descripción de lo ocurrido- que la comunica-

48 A la reunión asistieron: Thomas Bell, profesor de Zoología en el King’s College de Londres; John Jospeh Bennett, Banksian herbarium y biblioteca del British Museum; Sir Charles Lyell; el doctor Joseph Dalton Hooker; Mr. Nathaniel B. Ward; Mr. John M. Camplin; Mr. Robert Heward; el docotor Fredric D. Dyster; Mr. Daniel Oliver junior; Mr. Samuel P. Pratt; Mr. S. James A. Salter; Mr. William Archer; Mr. John Ball; Mr. John Thomas Syme; Mr Frederick Curry; el doctor William Baird, M.D. del British Museum; el doctor William Henry Fitton; Mr. Samuel Stevens, tesorero de la Entomological Society; el doctor William Benjamin Carpenter; el doctor Bethold Seemann; Mr. Arthur Henfrey, profesor of Botánica en el King's College de Londres; Mr. Benjamin W. Hawkins; el doctor William John Burchell; Mr. George B. Buckton; Mr. William M. Buckton; y Mr. Black, miembro asociado. El doctor Dyster llevó como invitado al doctor Baly, y Mr. Ward al doctor Melville. Tomo la relación de Moody, J.W.T., l.c., p. 474.

${ }^{49}$ L.c., p. 475. 
ción conjunta de Darwin y Wallace pasó poco menos que inadvertida. Frente al recuerdo emocionado de Hooker sobre lo sucedió aquella tarde — digno de recordarse como uno de los acontecimientos más destacados en la epopeya científica de la humanidad-, Moody admite que el 1 de julio de 1858 pudieron dar comienzo muchas cosas importantes para las ciencias de la vida, pero insiste, como he apuntado ya, en que "The actual event seems to be second only to the presentation of Mendel's discovery of the laws of genetics as an historical "nonevent" $"{ }^{50}$. ¿Es adecuado considerarlo así? Pienso que no, y que el silencio tras la lectura de los ensayos de Darwin y Wallace exige otra crónica.

En primer lugar, no puede concebirse que un auditorio tan selecto desde el punto de vista científico dejara de percibir el alcance de los textos de nuestros dos naturalistas, frente a comunicaciones como las de Dyster o Highley. Otra cosa es que despertaran la desaprobación o el rechazo de muchos de los presentes, pero la aparente indiferencia no pudo ser tal. Hooker comentaría más tarde a Francis Darwin: «... el interés suscitado fue intenso, pero el tema era demasiado nuevo y amenazador como para que la vieja escuela se alistara sin armarse antes ${ }^{51}$. Después de la reunión hubo una pequeña controversia — recordaba también-, pero el apoyo de Lyell y el suyo - declara con manifiesta autocomplacencia - intimidó a los socios, "pues de otro modo se hubieran abalanzado contra la teoría" ${ }^{52}$. Cabe, sin duda, que en el recuerdo de Hooker quede magnificado su protagonismo, pero lo cierto es que él y Lyell pidieron la palabra, antes de que los trabajos de Darwin y Wallace fuesen leídos, para enfatizar su enorme importancia ${ }^{53}$. Así pues, el que se presentaran los primeros, la entusiasta advertencia de Lyell y Hooker y el propio contenido de los ensayos hacen poco verosímil la descripción de Moody, de acuerdo con la cual los textos no lograron captar la atención de los treinta y dos miembros de la sociedad sentados en la sala ${ }^{54}$, y se perdieron entre los tediosos estudios examinados a lo largo de la prolongada reunión. Es un relato de lo que ocurrió desmentido doblemente, además, por el testimonio y la actitud de Bentham, el vicepresidente electo. También Bentham diría más tarde que

${ }^{50}$ L.c., p. 476.

51 Darwin, F. (ed.) 1887. The Life and Letters of Charles Darwin, Including an Autobiographical Chapter. London: John Murray, 2006, vol. II, p.126.

52 Ibid.

53 Véase Moody, J.W.T., l.c., p. 475.

${ }^{54}$ La Linnean Society contaba con 317 socios más. 
el interés de los asistentes fue vivo ${ }^{55}$, pero su modo de proceder es aún más revelador, pues tras escuchar el contenido de los documentos de Darwin y Wallace, quedó tan perturbado que retiró las «Notes on British Botany» — de inequívoco enfoque fijista-, enviadas ese mismo día a Bennett ${ }^{56}$. Con seguridad, no debió de ser el único impresionado por lo que escuchó. Todo invita a pensar que Hooker y Moody — cada uno desde su perspectiva — nos ofrecen una verdad a medias. La comunicación no pasó desapercibida desde luego, como Hooker, con justicia, narró después. Aun así, es cierto — según pretende Moody - que en el transcurso de la reunión no hubo la menor controversia, valoración o posicionamiento en torno a las hipótesis transformistas allí desarrolladas. Tal vez pueda atribuirse el silencio registrado al respeto que producía el padrinazgo de Lyell o a la necesidad de articular una respuesta antitransformista a la altura de los trabajos de Wallace y Darwin, pero se dieron dos causas más inmediatas que explican la aparente pasividad de los socios. La primera es que en aquellos días los miembros de la sociedad no recibían por adelantado ni los resúmenes ni tan siquiera los títulos de las comunicaciones que iban a ser leídas. Por lo tanto, ninguno de los presentes en la sesión tuvo noticia previa de que los ensayos de Darwin y Wallace iban a abrir el tiempo dedicado a la presentación de comunicaciones. La sorpresa fue, pues, absoluta, dejando poco margen a la reacción. En segundo lugar, la supuesta desidia de los asistentes — imaginada por Moody — desaparece en un sentido estricto, teniendo en cuenta que Thomas Bell —el presidente- no hizo ningún comentario tras la lectura de los textos ni permitió que se abriera un turno de réplica, para que pudieran realizarse observaciones u objeciones. Es más, Bentham señaló con posterioridad que Bell nunca habría consentido que alguien iniciase una discusión sobre un asunto presentado de forma tan inesperada ${ }^{57}$. Creo, en síntesis, que los tintes épicos con que Hooker elaboró el cuadro de lo ocurrido aquella tarde han de ser mitigados, si nos atenemos a la literalidad del libro de actas: ningún aplauso, ninguna crítica, ninguna observación, ningún comentario. No obstante, la callada recepción de los textos fue debida a su inesperado carácter, al prestigio de sus valedores, a la actitud de Bell y a la necesidad de diseñar una estra-

55 Véase la Introducción a Gardiner, B., MiLner, R. \& Morris, M. (eds.), 2008. Survival of the Fittest. A Special Issue of The Linnean celebrating the 150th anniversary of the Darwin-Wallace theory of evolution, Oxford: Wiley-Blackwell, p. 3.

56 Véase Gardiner, B., 1995. "The Joint Essay of Darwin and Wallace», The Linnean 1995 Volume (11) 1: 22.

57 Ibid. 
tegia opositora adecuada, pero nada tuvo que ver con el desinterés o la incapacidad de apreciar sus hondas implicaciones. El que Bentham retirase su trabajo así lo ratifica.

He comentado en las páginas anteriores que los Vestiges llegaron a ser atribuidos a Darwin. Es un dato que permite suponer que su inclinación hacia las tesis transformistas era, al menos en parte, conocida. Ahora bien, como he subrayado igualmente, el compromiso público no sólo con la realidad del proceso evolutivo sino con los precisos mecanismos que lo movilizan y en los que descansa resultaba algo completamente nuevo. La acelerada publicación de El origen de las especies el 24 de noviembre de 1859 - y el que ese mismo día se agotaran los 1.250 ejemplares de que constaba la edición — nos obliga a reconocer el interés de Darwin por dar a conocer sus ideas, la complicidad de Murray —el editor-, pero también, inequívocamente, que la obra se esperaba con inusitado interés. Cabe imaginar que los ecos de lo ocurrido en Burlington House debieron jugar algún papel en la actitud expectante de los lectores. Con todo, podría sostenerse que el revuelo suscitado por la aparición de El origen tuvo que ver con hechos no ligados a la reunión de julio de 1858 en la Linnean Society. Sin duda, en el clamoroso recibimiento de que el libro fue objeto pudieron intervenir múltiples factores. Aun así, no puede olvidarse que los textos de Darwin y Wallace se publicaron de forma inmediata —el 20 de agosto- en el Journal of the Proceedings of the Linnean Society. Hay aquí cierta asimetría ya respecto al tiempo que tardaron en publicarse los resultados de los experimentos de Mendel, pero concedamos que ésta sólo obedeciera a la diferente agilidad con que una y otra institución editasen sus actas. De cualquier manera, las consecuencias fueron muy distintas. Las actas de la Sociedad Linneana eran reseñadas con rapidez en numerosas publicaciones; entre ellas algunas de extraordinaria difusión como Athenaeum o Gardeners' Chronicle. Muy pronto - en octubre de ese año-, la Gardeners' Chronicle, que tenía entonces una tirada de 6.500 ejemplares, reprodujo el texto de Darwin leído el 1 de julio ${ }^{58}$. Las palabras escuchadas en el salón de sesio-

58 En la página 735 (No. 40, October 2nd, 1858), podía leerse: «The first part of the third volume of this important work attests to the determination of the Council to maintain the reputation of the Society as a great centre of scientific natural history. The zoological papers are varied and highly interesting; the botanical consists of further contributions to the orchidology of India as especially as relates to the genera Denobrium and Erica. Among others is a most suggestive paper by Mr. Darwin on variation among organic beings which we extract entire». Reproducido en GARDiner, B., Milner, R. \& Morris, M. (eds.), o.c., p. 3. 
nes tuvieron, así, con gran prontitud, miles de destinatarios, puesto que no fueron sólo accesibles a los aproximadamente trescientos cincuenta miembros de la sociedad con posibilidad de leer las actas. Por el contrario, el texto de Mendel dormiría en los archivos de la sociedad de Brno durante décadas. No es posible, en síntesis, hacer de la reunión en Picadilly una asamblea cuyo mayor significado histórico habría consistido en no tener ninguno. Existe una conexión decisiva y directa entre lo que sucedió el 1 de julio de 1858, la aparición de los ensayos de Darwin y Wallace en las actas de la sociedad, su divulgación en revistas con un eco importante y la preparación del ambiente en que sería recibido $E l$ origen de las especies. La flemática actitud general que acompañó a la recepción de los textos de ambos naturalistas queda justificada por la concurrencia de factores que he mencionado. Desde sus respectivas visiones, Hooker y Moody han ofrecido, a mi entender, un relato en exceso apasionado de lo que ocurrió o de lo que no ocurrió. Sea como fuere, doce días después —el 13 de julio—, Darwin expresaba con claridad cuál era su estado de ánimo ante el desarrollo de la sesión en Burlington House, cuando escribía al primero de ambos para decirle simplemente: "I am more than satisfied at what took place at the Linnean Society ${ }^{59}$.

\section{Bibliografía}

Burkhardt, F., Smith, S. Porter, D. et al. (eds.), 1985-2009. The correspondence of Charles Darwin, Cambridge: Cambridge University Press.

ChAmbers, R., 1844. Vestiges of the Natural History of Creation, Chicago: University of Chicago Press, 1994.

Claridge, M., 2008. «Alfred Russel Wallace - a Welsh Entomologist!», en Gardiner, B., Milner, R. \& Morris, M. (eds.). Survival of the Fittest. A Special Issue of The Linnean celebrating the 150th anniversary of the Darwin-Wallace theory of evolution, Oxford: Wiley-Blackwell, pp. 17-24.

Darwin, C. Esq., F.R.S., F.L.S.., \& F.G.S., and Wallace, A. Esq. Communicated by Sir Charles Lyell, F.R.S., F.L.S., and J.D. Hooker, Esq., M.D., V.P.R.S., F.L.S., \&c., 1858, «On the Tendency of Species to form Varieties; and on the Perpetuation of

59 DARWIN, F. (ed.) 1887. The life and letters of Charles Darwin, including an autobiographical chapter. London: John Murray, 2006, p. 128. La cursiva es de Darwin. 
Varieties and Species by Natural Means of Selection", Journal of the Proceedings of the Linnean Society, Zoology, 3: 45-62.

DARWIN, C., 1958. The autobiography of Charles Darwin 1809-1882. With the original omissions restored. Edited and with appendix and notes by his grand-daughter Nora Barlow, London: Collins.

DARWIN, F. (ed.) 1887. The life and letters of Charles Darwin, including an autobiographical chapter. London: John Murray, 2006.

Gardiner, B., 1995. «The Joint Essay of Darwin and Wallace», The Linnean 1995 Volume (11) 1: 13-24.

Gardiner, B., Milner, R. \& Morris, M. (eds.), 2008. Survival of the Fittest. A Special Issue of The Linnean celebrating the 150th anniversary of the Darwin-Wallace theory of evolution, Oxford: Wiley-Blackwell.

Herschel, J., 1830. A Preliminary Discourse on the Study of Natural Philosophy, Chicago-London: The University of Chicago Press, 1987.

MarChAND, J., 1916. Alfred Russel Wallace: Letters and Reminiscences, London-New YorkToronto-Melbourne: Cassell \& Company, 2005.

Moody, J.W.T., 1971. "The reading of the Darwin and Wallace papers: an historical "non event" ", Journal of the Society for the Bibliography of Natural History 5(6): 474476.

RuSE, M., 1979. The Darwinian revolution: science red in tooth and claw, Chicago: University of Chicago Press.

SARKAR, S., 2008. «Wallace and Natural Selection, 1858», Resonance: 236-244.

SCHWARTZ, J.S., 1990. «Darwin, Wallace, and Huxley, and Vestiges of the Natural History of Creation", Journal of the History of Biology, 23: 127-153.

WALLACE, A.R., 1855. «On the Law which has regulated the Introduction of New Species", Annals and Magazine of Natural History, 16 (2 ${ }^{\text {nd }}$ Series): 184-192.

WaLLACE, A.R., 1905. My Life: A Record of Events and Opinions, Whitefish (Montana): Kessinger Publishing, 2004.

Recibido: 25/08/2009

Revisado: 5/09/2009 